\title{
A dynamical model of growth of membership to an opinion
}

\author{
Prashant Goswami ${ }^{1, *}$ and Shivnarayan Nishad ${ }^{2}$ \\ ${ }^{1}$ Institute of Frontier Science and Applications, Bengaluru 560 037, India \\ ${ }^{2}$ CSIR National Institute of Science, Technology and Development Studies, Dr K.S. Krishnan Marg, New Delhi 110 012 , India
}

\begin{abstract}
Many social processes, from elections to terrorism, depend on growth of memberships to opinions. In a generic sense, an opinion is a proposition that for an individual has financial, cultural and emotional implications. The individual responses in turn create a 'social response' which influences the individual response resulting in a dynamical system with two-way feedbacks. We consider a set of deterministic dynamical equations that describe individual response to a class of prescribed opinions. The time-dependent opinion dynamics model exhibits nearly complete acceptance to nearly complete rejection with complex evolution, providing the framework for a mechanistic description of opinion formation.
\end{abstract}

Keywords: Dynamic model, growth of membership, opinion dynamics, social engineering.

ADVANCEMENT of many social processes can be visualized as evolution of growth of membership to a particular school of thought. In a social system, this membership grows through the response of individuals of the society to a given thought or proposition. Quantitative and causal understanding of the process of opinion formation (POF) can have multi-faceted applications. Besides, with growing computing power, it is now possible to consider simulation of responses of a large population to an opinion as a function of time ${ }^{1,2}$.

The complexity involved in opinion ${ }^{3-6}$ or consensus formation $^{7-9}$ has been noted and several studies have provided consistent or quantitative descriptions of opinion formation. An aspect that has received particular emphasis is the process/processes through which a large majority of people give up to an initial minority view ${ }^{10,11}$. These studies highlighted the importance of aggressiveness ${ }^{12}$ and persuasive power (of a minority) ${ }^{10,11}$ against larger numbers (majority) ${ }^{13}$ that are more passive. An important implication of such a view is that reforms are possible only using social violence or authoritarian top-leadership decisions ${ }^{14}$. Several studies have also considered social processes among microorganisms ${ }^{15}$ and animals ${ }^{16}$. It was recognized by several workers that certain aspects of the process of POF could be given dynamical descriptions ${ }^{17-20}$.

*For correspondence. (e-mail: siid.ifsa@gmail.com)
It is also clear that consensus formation ${ }^{7-9}$, or growth of opinion $^{3,4,6,20}$, can manifest itself in many forms of varying complexity, from animal herding ${ }^{21}$ to elections ${ }^{22,23}$ to strategic alliance ${ }^{23}$. The question of consensus among individuals was addressed early ${ }^{24}$, for example, the De Groot model addressed the question of emergence of consensus in a group of $k$ individuals with respect to a common subjective probability distribution. Berger ${ }^{24}$ explored a necessary and sufficient condition for such a De Groot's consensus. An empirical panhuman threshold that regulates man's organization of his natural and social environment was proposed in 1990 by $\mathrm{Kosse}^{25}$. He suggested that the thresholds were related to underlying regularities in the organization of long-term memory, and provided some hypotheses concerning group $\operatorname{size}^{25}$ and hierarchical complexity ${ }^{3}$. However, most works have concentrated on analysing the complex psycho-sociological mechanisms involved in the process of opinion forming ${ }^{26}$.

There have been several attempts in the mathematical modelling of opinion forming ${ }^{1-6}$. An important issue in POF within an interacting group is the development of consensus, polarization or fragmentation. A variety of techniques, both linear and nonlinear, have been applied to study this issue, such as matrix theory, Markov chain and graph theory. One of the social systems that has attracted interest of the mathematical modelling community is the election. In a sense, election campaigns are aimed at opinion formation, especially among undecided voters. It is possible, for example, to model the diffusion of a political opinion in a society in terms of the macroscopic manifestation of a wide spectrum of local exchanges between individuals in the society. A mathematical model for evaluating the expectation of the margin of votes to be received as a result of election campaigns was proposed by Belenky and King ${ }^{27}$. The model results could be expressed in terms of the minimum of the maximum function of the difference of two bilinear functions with one and the same first vector argument ${ }^{28}$. Similarly, the spread of two political parties (opinions) was modelled using an epidemiological approach with a nonlinear mathematical model ${ }^{28}$. The population was assumed to be constant and homogeneously mixed ${ }^{28}$, for which equilibria were shown to exist analytically.

Another important subject that has attracted modelling of POF is terrorism ${ }^{29,30}$. A mathematical model of insurgency 
was represented by Farley ${ }^{29}$ based on the spin states of individuals ('yes' and 'no' spin) of interacting individuals ('atoms'). Similarly, the emergence of social networks has provided added dimension to social dynamics ${ }^{3,19,31}$.

Friendly/hostile relationship, akin to follower or opposer of an opinion, can be also modelled as signed graph in a social network theory framework ${ }^{31}$. In such a formalism, individuals represent nodes of the network, while edge of positive (negative) weight represents friendliness (hostility or opposition). The social relationships between individuals influencing their opinions in the case of structurally balanced social networks have been well studied ${ }^{3,19,31}$. A two-party political system provides a classic example of opinion dynamics in a social system $^{28}$. Studies have been conducted to specify principles underlying how individuals are affected by their social environment. For example, computational models can be applied to simulate macro-level phenomena like change of attitude in a population based on micro-level (Latan's) theory ${ }^{32}$. In particular, such models could simulate a stable equilibrium of an incomplete polarization of opinions in which coherent minority subgroups could exist in the margins of population ${ }^{10,11}$.

Opinion formation also involves complex and often non-quantitative aspects like belief ${ }^{33}$, mutual understanding and persuasion ${ }^{34}$; growth of membership to an opinion also depends on processes like rumour transmission ${ }^{35}$. Dynamics of continuous opinions has been examined using analytical methods and computer simulations ${ }^{36}$, both static and time-dependent, as well as in the presence of nonlinearities with bounded confidence of the agents. Kawachi proposed deterministic models for transmission of rumour for the age-independent case $^{37}$, using a finitedimensional ordinary differential equation, with the solution converging to an equilibrium ${ }^{35}$. Essentially, the Kawachi model utilizes functional analysis and formulates the model as an abstract Cauchy problem on an infinite-dimensional Banach space. The results showed that, under certain assumptions, nontrivial equilibria exist. In such an approach, a system of $n$ experts is modelled as a positive discrete dynamical system in $n$ dimensions. One can then arrive at a sufficiency condition for reaching a consensus ${ }^{35}$. A theory describing the functioning of an individual in the presence of others was examined through computer simulation ${ }^{36}$.

An important aspect of opinion dynamics is group $\operatorname{size}^{4,25}$. While computational opinion dynamics was proposed as early as 1998 (ref. 2), several recent works have also suggested similar approaches ${ }^{4,6,10}$; computer simulations on binary opinion dynamics have been used in several works ${ }^{23,36}$. An important question is whether POF can be given a deterministic formulation that can lead to a causal, mechanistic description of the process.

We visualize POF as a dynamical process involving drivers and feedbacks that govern individual opinions; the membership to a given opinion changes as the number of individuals whose personal opinion is within a (fuzzy) ball $^{37}$ around the given opinion changes, thus resulting in a dynamical set of membership. The dynamical set is considered based on membership criteria. We consider a number of prescribed opinions to examine the response of the system.

\section{Methodology}

\section{The dynamical set of membership}

For a given population of $N_{I}$ individuals (assumed constant for simplicity; generalization is straightforward), we consider a membership set $\left(N_{\mathrm{M}}\right.$ is a set of members from the set of followers of an opinion in between lower and upper thresholds for the membership of the prescribed opinion) as

$$
\begin{aligned}
& N_{\mathrm{M}}(t)=\left\{x_{i}(t) \mid T_{\mathrm{L}} \leq x_{i}(t) \leq T_{\mathrm{H}}\right\}, \\
& x_{i}(t) \in\left(X_{\max }(t), X_{\min }(t)\right) .
\end{aligned}
$$

Here $x_{i}(t)$ is the opinion/response of the $i$ th member as a function of parameter $t$ (time). The number of members $\left(N_{\mathrm{M}}(t)\right)$ at time $t$ is the number of individuals out of the total members $\left(N_{\mathrm{T}}\right)$ who meet the above criterion at time $t$, or in other words, the member of the set of followers of an opinion. $T_{\mathrm{L}}$ and $T_{\mathrm{H}}$ respectively, represent the lower and upper thresholds for the membership of followers of the prescribed opinion. $X_{\max }(t)$ and $X_{\min }(t)$ respectively, represent the maximum and minimum opinion at time $t$.

Similarly, we define membership set for leftists $\left(N_{\mathrm{L}}(t)\right)$ and rightists $\left(N_{\mathrm{R}}(t)\right)$ as follows

$$
\begin{aligned}
& N_{\mathrm{L}}(t) \text { : individuals for whom } x_{i}(t)<T_{\mathrm{L}}, \\
& N_{\mathrm{R}}(t) \text { : individuals for whom } x_{i}(t)>T_{\mathrm{H}} .
\end{aligned}
$$

The lower and upper thresholds for membership of followers can be defined as

$$
T_{\mathrm{L}}=x_{0}\left(1-\varepsilon_{\mathrm{L}}\right), \quad T_{\mathrm{H}}=x_{0}\left(1+\varepsilon_{\mathrm{H}}\right) .
$$

The opinion $x_{0}$ and an individual opinion/response $x_{i}(t)$ belong to the same vector space, except that $x_{0}$ is not assigned to any particular member; rather it drives an individual opinion $x_{i}(t) . \varepsilon_{\mathrm{L}}$ and $\varepsilon_{\mathrm{H}}$ respectively, represent the coefficient of strength for the lower and upper thresholds of opinion $x_{0}$.

While $N_{\mathrm{M}}(t), N_{\mathrm{L}}(t)$ and $N_{\mathrm{R}}(t)$ are sets of membership defined by eqs (1)-(3), we shall also designate the number of members in each of these sets by the corresponding symbol for ease of discussion. Thus, for example, $N_{\mathrm{M}}(t)$ also designates the number of members in the set $N_{\mathrm{T}}$.

An individual $i$ is a member (follower) of opinion $x_{0}$ if it satisfies the condition

$$
T_{\mathrm{L}} \leq x_{i}(t) \leq T_{\mathrm{H}}
$$




\section{The dynamical model}

The equation describing the dynamics of the $i$ th individual opinion is given by

$$
\begin{aligned}
\frac{\mathrm{d} x_{i}}{\mathrm{~d} t}=- & \alpha\left|x_{i}(t)-x_{0}(t)\right|+\beta * N_{\mathrm{M}}\left|x_{0}(t)\right| \\
& -\gamma\left(N_{\mathrm{T}}-N_{\mathrm{M}}(t)\right)\left|x_{0}(t)-\bar{x}(t)\right| \\
& +\delta\left|x_{i}(t)-x_{\mathrm{G}}\right|+\rho\left|\bar{x}(t)-x_{\mathrm{G}}\right|+a_{\mathrm{R}} * x_{\mathrm{R}}(t) .
\end{aligned}
$$

The first term on the right-hand side of eq. (5) represents restoration of the individual opinion $x_{i}(t)$ to the stagnant opinion $x_{0}(t)$, with a strength characterized by the parameter $\alpha$. The second term represents a forcing on the individual opinion $x_{i}(t)$ due to rise in membership of the opinion at time $t\left(N_{\mathrm{M}}(t)\right)$ and strength of this forcing characterized by the parameter $\beta$. The third term represents the effect due to opposition to $x_{0}$ from the remaining individuals $\left(N_{\mathrm{T}}-N_{\mathrm{M}}\right)$, and is assumed proportional to the unorthodoxy of $x_{0}$. The term involving $\delta$ represents a restoration of the individual opinion to the given global opinion $\left(X_{\mathrm{G}}\right)$, while the term involving $\rho$ represents a restoration of the average of the individual opinions $\left(x_{0}(t)\right)$ to the global opinion.

$x_{\mathrm{R}}(t)$ represents a random component in the dynamics of each individual at every time step and is represented as

$$
x_{\mathrm{R}}(t)=1-2 \times r(t) \text {, such that } x_{\mathrm{R}}(t) \in(-1,1),
$$

where $r(t)$ is a random number between -1 and 1 .

We adopt the initial condition that represents a random distribution of opinions among the individuals.

$$
x_{i}(t=0)=1-2 \times r(t) \text {, such that } x_{i}(t=0) \in(-1,1) .
$$

Initial conditions are similar for all types of opinions and scenarios. The random initial condition is used as a canonical representation of the situation where no prior knowledge of the initial state exists; the limits $(-1,1)$ ensure that the states are bounded and normalized.

We assume the global opinion to contain contributions from the average opinion and the prescribed opinion; the part of the global opinion that is independent of $\bar{x}(t)$ and $x_{0}(t)$ is not considered here

$$
x_{\mathrm{G}}=C_{\mathrm{G} 1} * x_{0}(t)+C_{\mathrm{G} 2} * \bar{x}(t),
$$

where $C_{\mathrm{G} 1}$ and $C_{\mathrm{G} 2}$ respectively, represent the coefficients of global opinion from the prescribed opinion and average opinion.

As discussed above, group size plays an important role in social dynamics ${ }^{4,24}$. We shall adopt a minimum group size that exhibits reasonable behaviour in the opinion $x_{0}$. The minimum group size was adopted by considering a number of choices that led to bounded and/or convergent evolution.

\section{Representation of opinion and logical expectation}

In the absence of observed data for such a system, we use expected logical behaviour to calibrate and benchmark our results. We have assumed four types of opinions in the present study, which are described below.

\section{Average opinion}

The average opinion of the group and average opinion of members of the set of followers at any time $t$ can be defined by eqs (9) and (10) respectively

$$
\begin{aligned}
& x_{0}(t)=\bar{x}(t)=\frac{1}{N_{\mathrm{T}}} \sum_{i=1}^{N_{\mathrm{T}}}\left|x_{i}(t)\right|, \\
& \bar{X}_{\mathrm{M}}(t)=\frac{1}{N_{\mathrm{M}}} \sum_{i=1}^{N_{\mathrm{M}}}\left|x_{i}(t)\right|,
\end{aligned}
$$

where $\bar{x}(t)$ and $\bar{X}_{\mathrm{M}}(t)$ respectively, represent the average opinion of the group $\left(N_{\mathrm{T}}\right)$ and average opinion of members of the set of followers $\left(N_{\mathrm{M}}(t)\right)$. Thus eq. (9) provides specific representations of $x_{0}(t)$ introduced in eq. (8).

Since the opinion is an average of individual opinions, the logical expectation is that the membership will be nearly $100 \%$, with very few and nearly equal number in either extreme left or extreme right.

\section{Stagnant opinion}

We consider two other cases to understand the behaviour of the system. The first is that of a static opinion, fixed at the initial time; we express this as $x_{\mathrm{os}}=x_{i}(t=0)$ representing an extreme orthodox and conservative society with opinion fixed for all time.

The stagnant opinion is represented by the average of the initial individual opinions

$$
x_{0}(t)=\bar{x}_{I}=\frac{1}{N_{\mathrm{I}}} \sum_{i=1}^{N_{\mathrm{I}}} x_{i}(t=0),
$$

where $N_{\mathrm{I}}$ is the number of members following the stagnant opinion.

\section{Random opinion}

The other one is that of driving opinions random in time; this is expressed as $x_{\mathrm{or}}(t)=x_{i}(t)$, where $x_{i}(t)$ is the opinion of the $i$ th member chosen at random. 
Table 1. Parameters values for standard case (NM 100\%) for different opinion vectors under three scenarios: scenario 1 (SC1), scenario 2 (SC2) and scenario 3 (SC3)

\begin{tabular}{|c|c|c|c|c|c|c|c|c|c|c|c|c|c|c|c|c|}
\hline \multirow{2}{*}{ Parameter } & \multirow{2}{*}{ Standard } & \multicolumn{15}{|c|}{ Opinion $\left(10^{-4}\right)$} \\
\hline & & \multicolumn{3}{|c|}{$x_{0}=\bar{x}(t)$} & \multicolumn{3}{|c|}{$x_{0}=X_{\min }$} & \multicolumn{3}{|c|}{$x_{0}=X_{\max }$} & \multicolumn{3}{|c|}{$x_{0}=\bar{x}_{\mathrm{I}}$} & \multicolumn{3}{|c|}{$x_{0}=R(t)$} \\
\hline$\varepsilon_{\mathrm{L}}$ & 0.9 & - & - & & - & - & & - & - & & - & - & & - & - & \\
\hline$\varepsilon_{\mathrm{H}}$ & 0.9 & - & - & & - & - & & - & - & & - & - & & - & - & \\
\hline$\alpha$ & 0.000007 & 0.5 & 0.004 & & 0.001 & 0.009 & & 0.001 & 0.003 & & 0.001 & 0.009 & & 0.01 & 0.05 & \\
\hline$\beta$ & 0.00017 & - & - & & - & - & & - & - & & - & - & & - & - & \\
\hline$\gamma$ & 0.0000025 & - & - & & - & - & & - & - & & - & - & & - & - & \\
\hline$\delta$ & 0.0022 & - & - & & - & - & & - & - & & - & - & & - & - & \\
\hline$\rho$ & 0.000081 & - & - & & 0.0004 & 0.003 & & - & - & & 0.002 & 0.002 & & 0.002 & 0.002 & \\
\hline$C_{\mathrm{G} 1}$ & 5.0 & - & - & & - & - & & - & - & & - & - & & - & - & \\
\hline$C_{\mathrm{G} 2}$ & 5.0 & - & - & & - & - & & - & - & & - & - & & - & - & \\
\hline
\end{tabular}

For an opinion that is random in time, we expect very small or zero membership, although this opinion would be within the range of $x_{\mathrm{i}}(t)$.

$$
x_{0}(t)=x_{\mathrm{or}}(t)=1-2 \times r(t)
$$

where $r(t)$ is a random number between 0 and 1 , and the values of $x_{\mathrm{or}}(t)$ lies between -1 to 1 . The random number is generated from the Fortran 77 library function.

\section{Extremist and extreme opinions}

We define extremist opinions as averages over the rightists $\left(N_{\mathrm{R}}\right)$ and the leftists $\left(N_{\mathrm{L}}\right)$

$$
\begin{aligned}
& x_{\mathrm{or}}(t)=\frac{1}{N_{\mathrm{R}}} \sum_{i=1}^{N_{\mathrm{R}}} x_{i}(t), \text { such that } x_{i}(t)>T_{\mathrm{H}}, \\
& x_{\mathrm{or}}(t)=\frac{1}{N_{\mathrm{L}}} \sum_{i=1}^{N_{\mathrm{L}}} x_{i}(t), \text { such that } x_{i}(t)>T_{\mathrm{L}} .
\end{aligned}
$$

Here $T_{\mathrm{L}}$ and $T_{\mathrm{H}}$ are the lower and upper thresholds for membership of the followers of an opinion.

In addition, we consider two extreme opinions in terms of the maximum and minimum values of $x_{i}(t)$ at given time $t$. Thus,

$$
\begin{aligned}
& x_{0}(t)=x_{0(\min )}(t)=\min \left\{x_{i}(t)\right\}, \\
& x_{0}(t)=x_{0(\max )}(t)=\max \left\{x_{i}(t)\right\} .
\end{aligned}
$$

Here $x_{0(\max )}(t)$ and $x_{0(\min )}(t)$ respectively, represent the maximum and minimum opinions of the group of opinions.

\section{Calibration and benchmark state}

In the absence of constitutive relations or theoretical/observation constraints, the set of parameters describ- ing the system (Table 1) cannot be ascribed unique values. Instead, our approach is to consider a set of values that produces 'reasonable' behaviour and examines the system dynamics against this reference state due to various processes. Thus although the parameters can, in principle, be arbitrary, they are only varied around their calibrated values to study their impact.

An important parameter in opinion dynamics is the size of the group, and several studies have emphasized the importance of group size. A review of some models in the social sciences in which system size plays an important role in the final outcome of the dynamics was presented by Toral and Tessone ${ }^{4}$. Some of these models examined the conditions under which changes in behaviour can appear only when the number of agents in the model takes a finite value; such changes in behaviour can be related to the apparent phase transitions that appear in some physical models. In our case, group size affects parameters like average opinion/response. The system was calibrated so that for the standard set of parameters, it produced membership close to $100 \%$ for an opinion expressed as the average of individual opinions. Based on the general system behaviour, discussed below, a standard set of parameters was adopted for analysis.

\section{Results}

The behaviour of the system has been studied under different initial distributions as well as varying strengths and combinations of the restoring forces.

\section{Effect of random forcing on the evolution of POF}

It was found that without a random component, however small, in the dynamics (last term in eq. (5)), the evolution of membership did not show much variation in time; the corresponding average and maximum of individual opinions showed nearly monotonic increase (Figure 1, right column). This was found to be true for different 

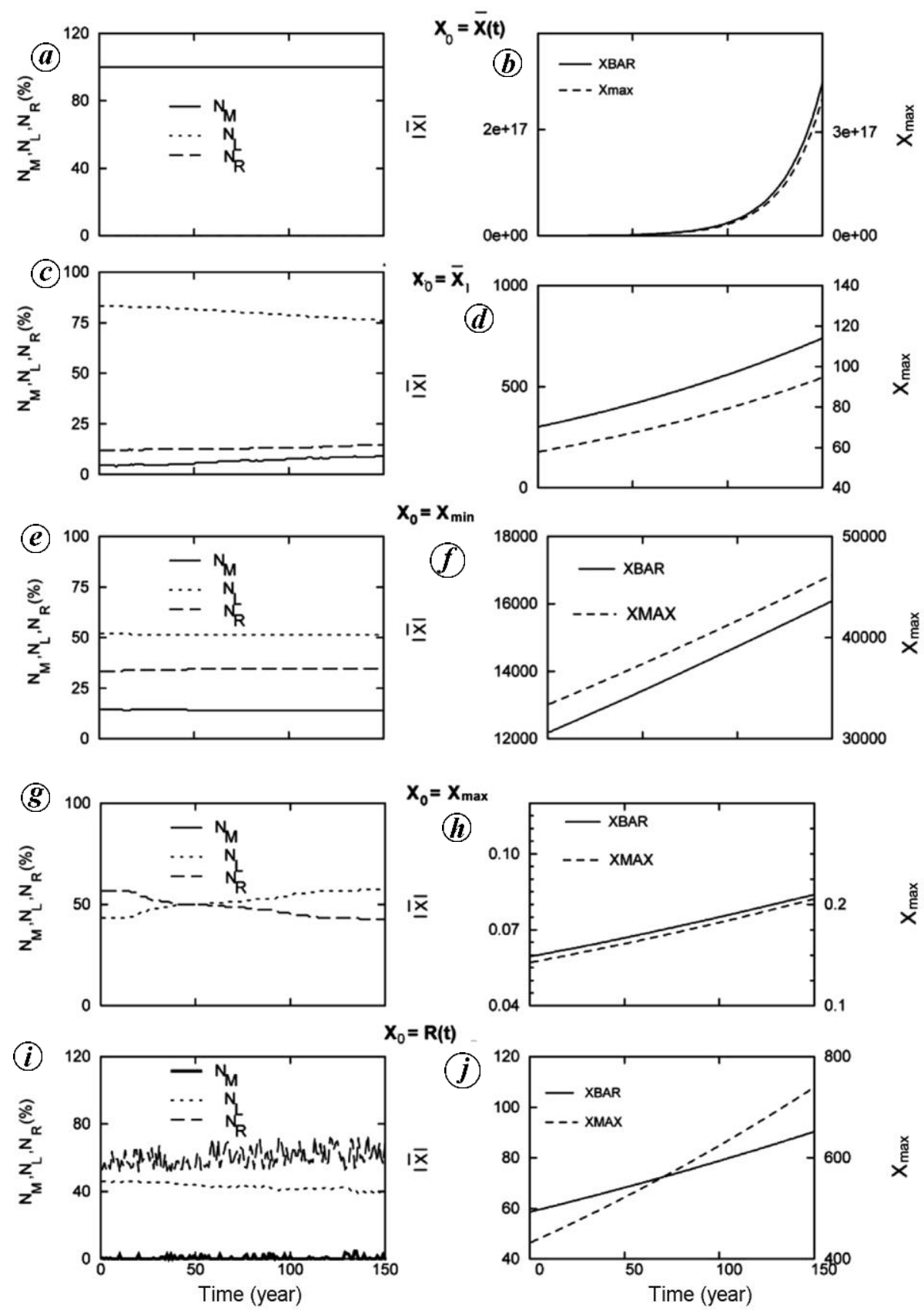

Figure 1. Evolution of membership and characteristics of five representations (average, stagnant, minimum, maximum and random opinions) of the prescribed opinion without random forcing $(\operatorname{ar}=0.0)$ in the dynamics. (Left) Growth of members $\left(N_{\mathrm{M}}(t)\right.$, solid line), extreme leftists $\left(N_{\mathrm{L}}(t)\right.$, dotted line) and extreme rightists $\left(N_{\mathrm{R}}(t)\right.$, long dashed line) as percentage of the total number (150). (Right) Average $\left(\bar{x}\right.$, left $y$-axis, solid line) and maximum of individual opinions ( $X_{\max }$, right $y$-axis, dashed line). Values of the parameters correspond to the standard set. $a-j$ represent the subplots of the figure.

representations of the prescribed opinions. In contrast, introduction of even a small random component in the dynamics resulted in complex, non-trivial dynamics for each of the five representations of the prescribed opinion (Figure 2). The membership for average opinion without random component was about $100 \%$ (Figure $1 a$ ), while with random component it was about $80 \%$ (Figure $2 a$ ). Similarly, there was a major impact of the random com- ponent on POF in case of stagnant opinion, maximum opinion, minimum opinion and random opinion (Figures $1 b-e$ and $2 b-e)$.

While the presence of a random driving component is essential for non-trivial dynamics, its relative magnitude can be quite small throughout the dynamics. For example, for the standard case subsequently described, five of the six (non-random) terms in eq. (5) are comparable with a 

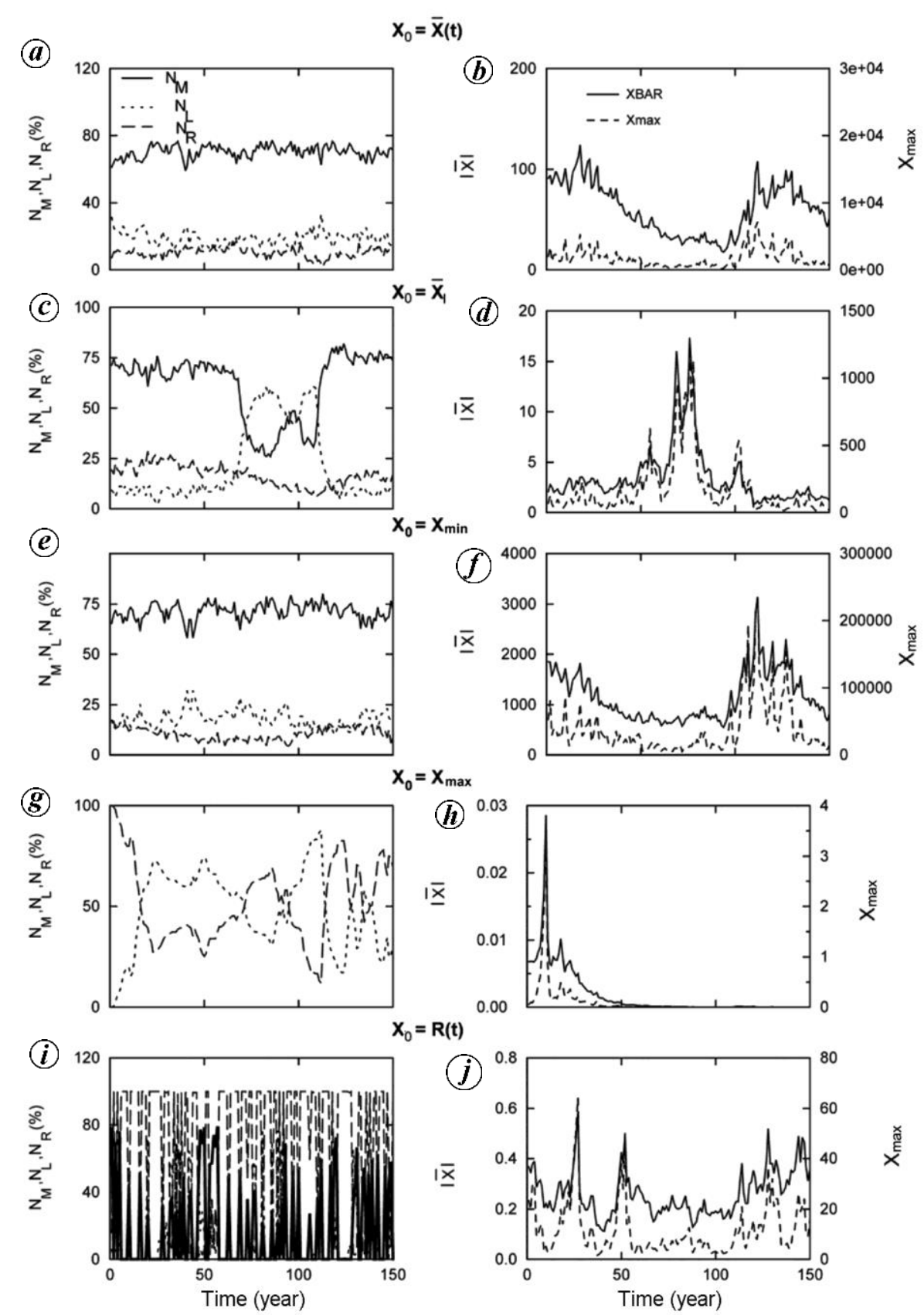

Figure 2. Evolution of membership and characteristics of prescribed opinion expressed for standard case as five scenarios: average individual opinions at time $t(\bar{x}(t))$, average of the individual opinions at the initial time $\left(\bar{x}_{\mathrm{I}}(t)\right)$, minimum and maximum of the random opinion $\left(X_{0}(t)=R(t)\right)$. (Left) Growth of members $\left(N_{\mathrm{M}}(t)\right.$, solid line $)$, extreme leftists $\left(N_{\mathrm{L}}(t)\right.$, dotted line $)$ and extreme rightists $\left(N_{\mathrm{R}}(t)\right.$, long dashed line) as percentage of the total number (150). (Right): Average ( $\bar{x}$, left $y$-axis, solid line) and maximum of individual opinions $\left(X_{\max }\right.$, right $y$-axis, dashed line). Values of the parameters correspond to the standard set. $a-j$ represent the subplots of the figure.

much smaller but non-zero random term (Figure 3). Thus the results below are not driven by the random component as the major forcing.

The random component in the dynamics of individual opinions represents various unsystematic effects. While we consider a random component in the dynamics unavoidable, the strength of the random component has appreciable effect on the evolution of the membership. It was found that the membership was close to $100 \%$ for random component equal to zero, but with undesirable behaviour of $\bar{x}$ and $X_{\max }$ (Figure $4 a$ and $d$ ). For much larger values of $a_{\mathrm{R}}(>1.2)$, on the other hand, the membership fails to approach $100 \%$ (Figure $4 b$ and $c$ ). It was found that a moderate value $\left(a_{\mathrm{R}}=0.9\right)$ provides 
membership close to $90 \%$ with acceptable behaviour of $\bar{x}$ and $X_{\max }$ (Figure $4 e$ and $f$ ); our subsequent discussions are based on a value of $a_{\mathrm{R}}=0.9$.

\section{Response to average opinion}

For the standard set of parameters (Table 2) with the amplitude of the random component $\left(a_{\mathrm{R}}\right)$ being 0.9 and the opinion $x_{0}(t)$ represented by the average opinion $\bar{x}(t)$, a situation exists for which the membership is $80 \%$ (Figure $5 a-c)$, with small membership in the extreme left or extreme right. The initial membership starts below $80 \%$, and then rises and stays close to it (Figure $5 \mathrm{a}$ ). The corresponding average and maximum opinion exhibit complex but bounded evolution (Figure $5 d$ ). This behaviour is fairly robust in the sense that it does not change drastically for different scenarios represented in terms of different values of $\alpha$ (Figure $5 b-f$ ). This set of parameters then provides the benchmark set against which we shall consider relative roles of various processes as well as response of the system to different representations of an opinion.

\section{Role of group size}

The dynamics of the opinion is also expected to depend on the number of individuals. Based on simulations for different values of $N_{\mathrm{I}}$, it was found that up to $N_{\mathrm{I}}=50$, membership for the standard case did not generally approach $100 \%$ (Figure $6 a$ ). However, beyond $N_{\mathrm{I}}=50$, the membership was found to be essentially independent

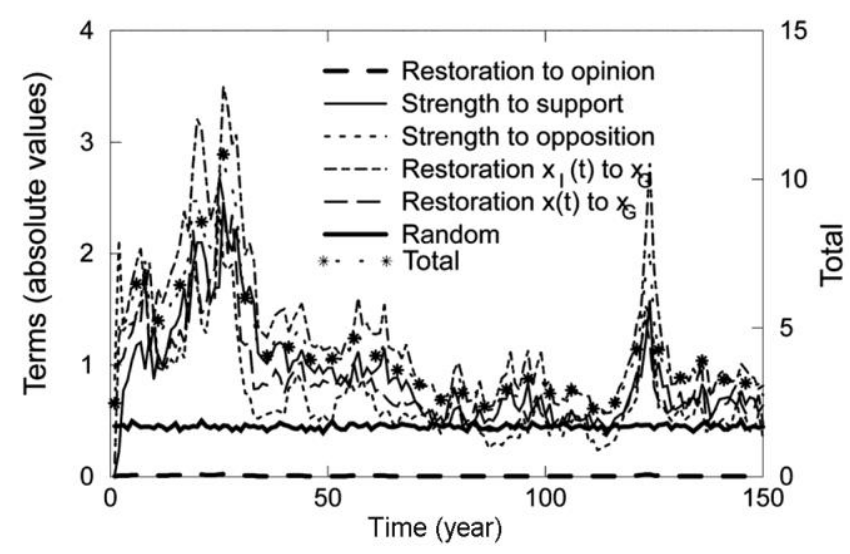

Figure 3. Absolute values of the terms in eq. (5) (left $y$-axis) for the standard case. The restoration of individual opinion to stagnant opinion (thick, long dashed line, left $y$-axis), strength of forcing on prescribed opinion due to rise of the membership of opinion (thin solid line, left $y$-axis), strength of opposition to the prescribed opinion $x_{0}$ from the remaining individuals (thin dashed line, left $y$-axis), restoration of individual opinion to global opinion (thick dashed line, left $y$-axis), restoration of average opinion to global opinion (thin, long dashed line, left $y$-axis) and random component in the dynamics of individual opinion (thick solid line, left $y$-axis) as a function of time. The total (sum of all terms) is given in the right $y$-axis (dotted line with stars). of the number of individuals (Figure $6 b-d$ ). The evolution of membership was found to be sensitive to larger values of $N_{\mathrm{I}}$. At $N_{\mathrm{I}}=500$, the membership was dominated by leftist, with less than $25 \%$ of the individuals supporting the prescribed opinion (Figure $6 c$ ). At $N_{\mathrm{I}}=600$, there were strong out-of phase oscillations up to $N_{\mathrm{I}}=150-500$ among the leftists and rightists, with no membership to the prescribed opinion (Figure $6 d$ ). We have therefore adopted a value of $N_{\mathrm{I}}=150$ in the subsequent discussions.

\section{Response to stagnant opinion}

For the stagnant opinion based on average of the initial individual opinions $\left(x_{0}(t)=\bar{x}_{\mathrm{I}}\right)$, the membership remained close to $75 \%$ up to about 50 years for the standard set of parameters (Figure $7 a$ ), with bounded $\bar{x}$ and $X_{\max }(t)$ (Figure $7 d$ ). This value in membership of the opinion then showed a steep decline (around the 60th year), with a sharp recovery around the 125th year (Figure $7 a$ ). This decline and rise in $N_{\mathrm{M}}(t)$ were similar but out of phase to changes in $N_{\mathrm{L}}(t)$ (Figure $7 a$ ). For the stagnant opinion, membership to extreme rightists stayed small $(<25 \%)$ throughout. These results also hold for different scenarios (Figure $7 b$ and $c$ ); for all these cases $\bar{x}$ and $X_{\max }(t)$ remain bounded (Figure $7 e$ and $f$ ).

\section{Response to extreme opinion}

Consistent with our formalism and expectation, no membership to the prescribed opinion evolved for opinions represented by $X_{\max }$ (eq. (13)); the membership belonged to either extreme left $\left(N_{\mathrm{L}}(t) \sim 60 \%\right)$ or extreme right $\left(N_{\mathrm{R}}(t) \sim 40 \%\right.$ ), with $N_{\mathrm{M}}(t) \sim 0$ (Figure $8 a$ ). However, complex dynamics of cross-over from extreme left to extreme right was also seen (Figure $8 b$ and $c$ ). For very strong restoration, the values of $N_{\mathrm{L}}(t)$ and $N_{\mathrm{R}}(t)$ began to converge (Figure $8 c$ ).

The situation driven by the opinion corresponding to minimum of the individual opinions $\left(X_{\min }\right)$ resulted in very different situations (Figure $9 a-c$ ). For weak restoration ( $\alpha=0.00005)$, membership to the prescribed opinion $\left(X_{\min }\right)$ stayed close to $75 \%$, but with strong variability in time (Figure $9 a$ ). Once again (as in Figure $8 a$ ), the variability in $N_{\mathrm{M}}(t)$ was opposite in phase to $N_{\mathrm{L}}(t)$, with $N_{\mathrm{R}}(t) \sim 0$ (Figure $9 a$ ). For stronger restoration ( $\alpha=$ $0.0001), N_{\mathrm{M}}(t)$ remained close to $75 \%$ throughout, with nearly equal values $(\sim 10 \%)$ of $N_{\mathrm{L}}(t)$ and $N_{\mathrm{R}}(t)$ to the prescribed opinion. Interestingly, the general nature of evolution of average opinion and maximum opinion was quite different for the three scenarios (Figure $9 d-f$ ).

\section{Response to random opinion}

For an opinion that is a random function of time $\left(X_{0}(t)=R(t)\right)$, the membership showed a highly variable 

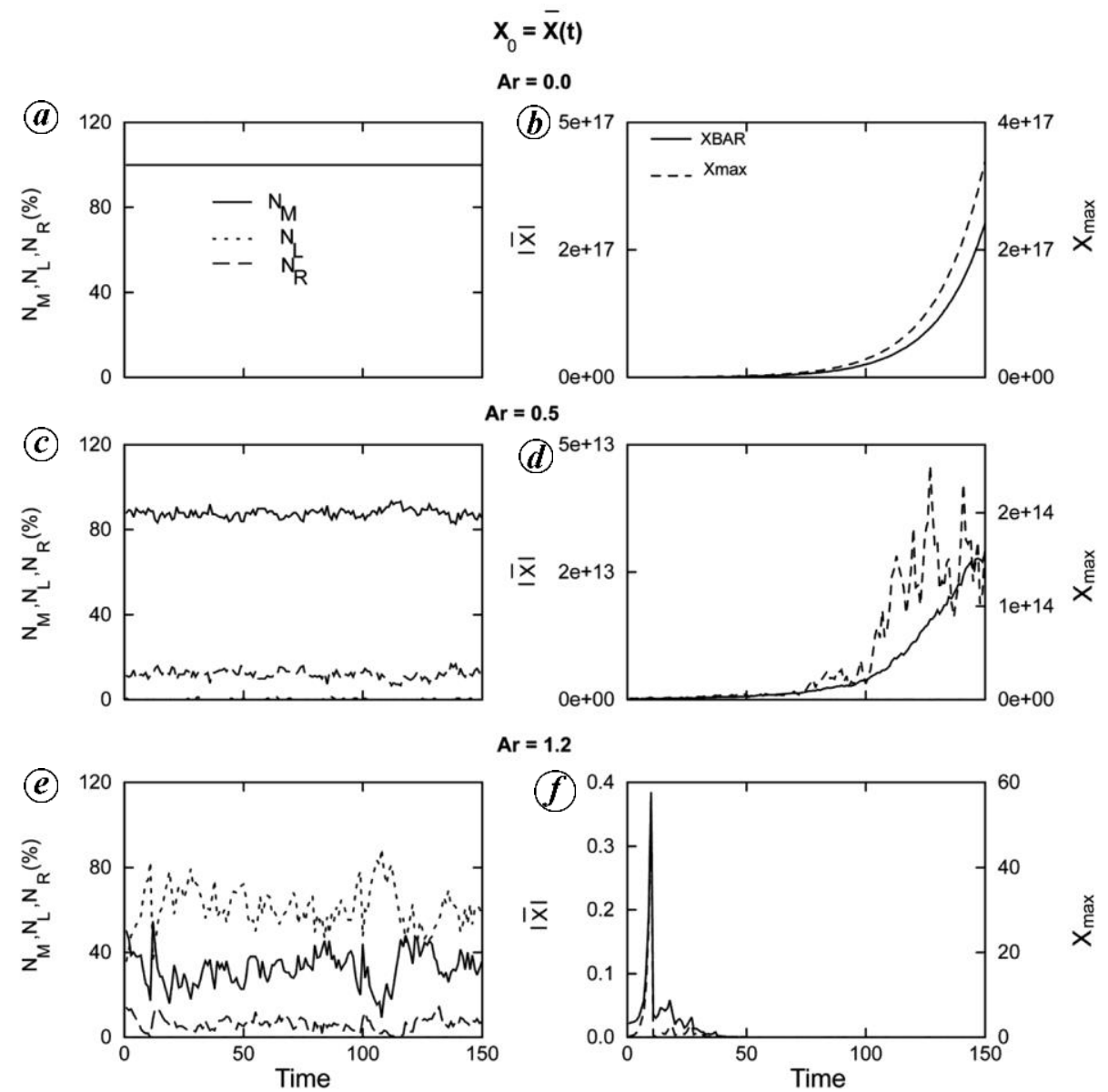

Figure 4. Impact of strength of random component in the evolution of membership of a prescribed opinion. (Left) Growth of members $\left(N_{\mathrm{M}}(t)\right.$, solid line), extreme leftists $\left(N_{\mathrm{L}}(t)\right.$, dotted line) and extreme rightists $\left(N_{\mathrm{R}}(t)\right.$, long dashed line) as percentage of the total number (150). (Right) Average ( $\bar{x}$, solid line, left $y$-axis) and maximum of individual opinions ( $X_{\max }$, dashed line, right $y$-axis). Values of the parameters correspond to the standard set. $a-f$ represent the subplots of figure.

Table 2. Values of parameters for standard opinion

\begin{tabular}{|c|c|c|c|}
\hline Process & Symbol & Standard value & Range considered \\
\hline Total population & $N_{\mathrm{I}}$ & 150 & $50-10,000$ \\
\hline Coefficient of restoration to the opinion & $\alpha$ & 0.00007 & $0.00000005-0.005$ \\
\hline Coefficient of strength of support to the opinion & $\beta$ & 0.00017 & $0.0000003-0.0007$ \\
\hline Coefficient of strength of opposition to the opinion & $\gamma$ & 0.0000025 & - \\
\hline Restoration of individual opinion to global opinion & $\delta$ & 0.0022 & $0.000009-0.01$ \\
\hline Restoration of average to global opinion & $\rho$ & 0.000081 & $0.00000001-0.01$ \\
\hline Threshold for membership: lower & $\varepsilon_{\mathrm{L}}$ & 0.9 & $0.5-1.2$ \\
\hline Threshold for membership: higher & $\varepsilon_{\mathrm{H}}$ & 0.9 & $0.5-1.2$ \\
\hline Coefficient of contribution of opinion to global opinion & $C_{\mathrm{G} 1}$ & 5.0 & $0.7-20.0$ \\
\hline Coefficient of contribution of average opinion to global opinion & $C_{\mathrm{G} 2}$ & 5.0 & $0.7-20.0$ \\
\hline Coefficient of random forcing of individual opinion & $a_{\mathrm{R}}$ & 0.9 & $0-1.5$ \\
\hline
\end{tabular}

behaviour, changing from nearly $100 \%$ leftists to close to $100 \%$ rightists for the standard case (Figure $10 a$ ). This behaviour was found to persist even for greater strength of restoration ( $\alpha=0.00005)$ to the prescribed (random) opinion (Figure $10 b$ and $c$ ). For all the three scenarios, the average $(\bar{x})$ and maximum $\left(X_{\max }\right)$ were found to exhibit complex dynamics with bounded values (Figure $10 d-f)$.

\section{Response to different processes}

Very interesting behaviour was found to result through variation of $\alpha$; while smaller values of $\alpha(<0.00009)$ resulted in slow growth in $N_{\mathrm{M}}(t)$ from about $50 \%$, larger values of $\alpha(\sim 0.0002)$ resulted in large values of $N_{\mathrm{L}}(t)$ and $N_{\mathrm{R}}(t)$ in the beginning, with small $N_{\mathrm{M}}(t)(\sim 15 \%)$ which may grow to only about $50 \%$. Thus for very strong 

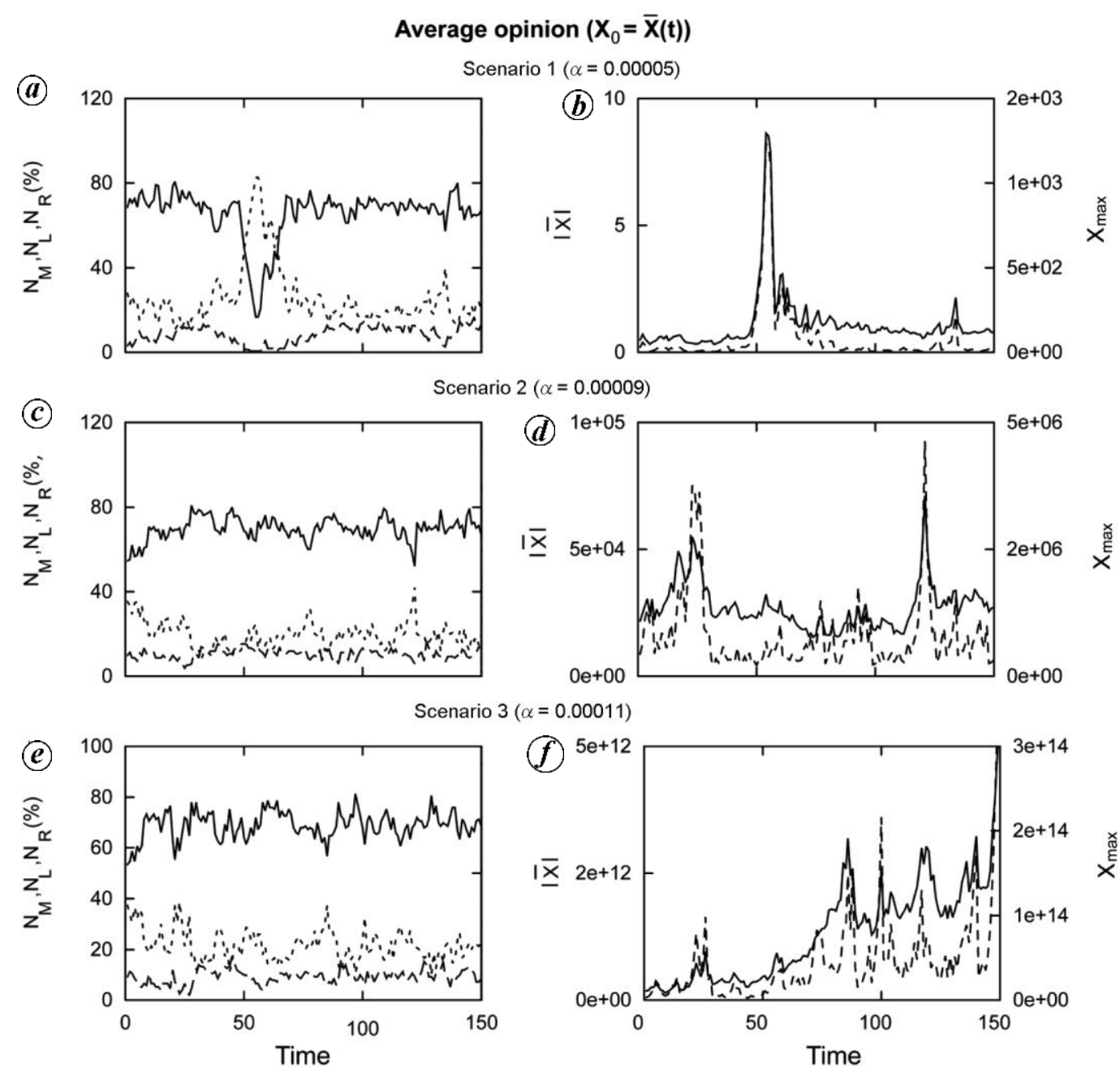

Figure 5. Evolution of membership and characteristics of prescribed opinion expressed as an average of individual opinions as a function of time. (Left) Growth of members $\left(N_{\mathrm{M}}(t)\right.$, solid line), extreme leftists $\left(N_{\mathrm{L}}(t)\right.$, dotted line) and extreme rightists $\left(N_{\mathrm{R}}(t)\right.$, long dashed line) as percentage of the total number (150). (Right) Average ( $\bar{x}$, solid line, left $y$-axis) and maximum of individual opinions ( $X_{\max }$, dashed line, right $y$-axis). Results are shown for three different scenarios. Values of the parameters correspond to the standard set. $a-f$ represent the subplots of figure.

restoration to the average opinion (a strictly consensusbased system), membership can become very small and fall below the extremist opinion (Figure 11). Small values of restoration to global opinion $(\rho<0.0001)$ resulted in membership that was stable around $80 \%$, with nearly equal values of $N_{\mathrm{L}}$ and $N_{\mathrm{R}}$ (Figure 11); the results were comparable to the standard case (Figure $11 \mathrm{~b}$ ). However, for much larger values of $\rho(\sim 0.001)$, the effects were dramatically different; $N_{\mathrm{M}}(t)$ decreased quickly to less than $40 \%$, while $N_{\mathrm{L}}$ and $N_{\mathrm{R}}(t)$ attained nearly equal values of $20 \%$ (Figure 11). The dynamics of the opinion was found to be not very sensitive to the strength of the opposition characterized by $\beta$ (Supplementary Figure 1 ). Similarly, the strength of restoration of the individual opinion to the global opinion $(\delta)$ was not found to have appreciable effects on the dynamics of the membership (Figure 12). With our representation of global opinion in terms of the average opinion and prescribed opinion, dynamics of the membership was found to be essentially independent of the structure of the global opinion (Supplementary Figure 2).

A summary of the response to different prescribed opinions in terms of $N_{\mathrm{M}}(t), N_{\mathrm{L}}(t)$ and $N_{\mathrm{R}}(t)$ in Table 3 shows that a wide spectrum of evolutions is possible with different prescribed opinions. At one end of this spectrum is the prescribed opinion represented by average of individual opinions, with high and nearly steady membership $\left(N_{\mathrm{M}}(t)\right)$ as expected. At the other end of the spectrum is the prescribed opinion random in time, for which no membership develops.

\section{Discussion and conclusion}

Quantitative and causal models of social processes are challenging, but can be of diverse and critical use. Essentially all major social processes, be it an organization of 


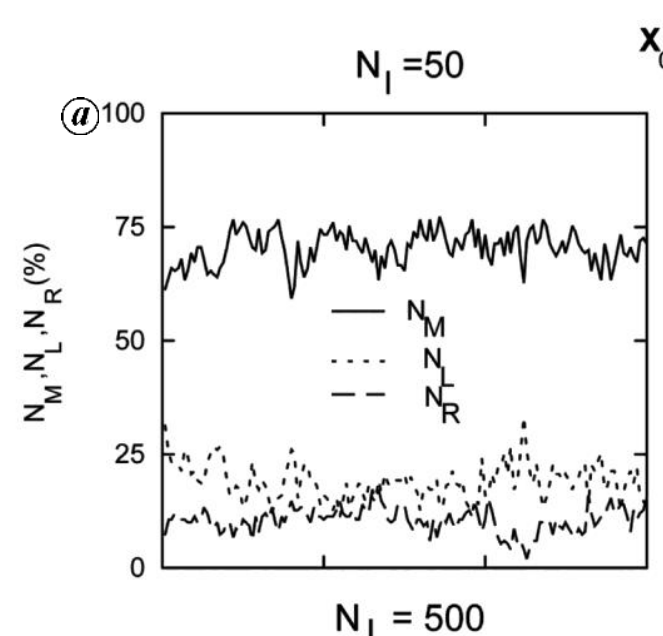

$\mathbf{X}_{0}=\overline{\mathbf{X}}(\mathbf{t})$
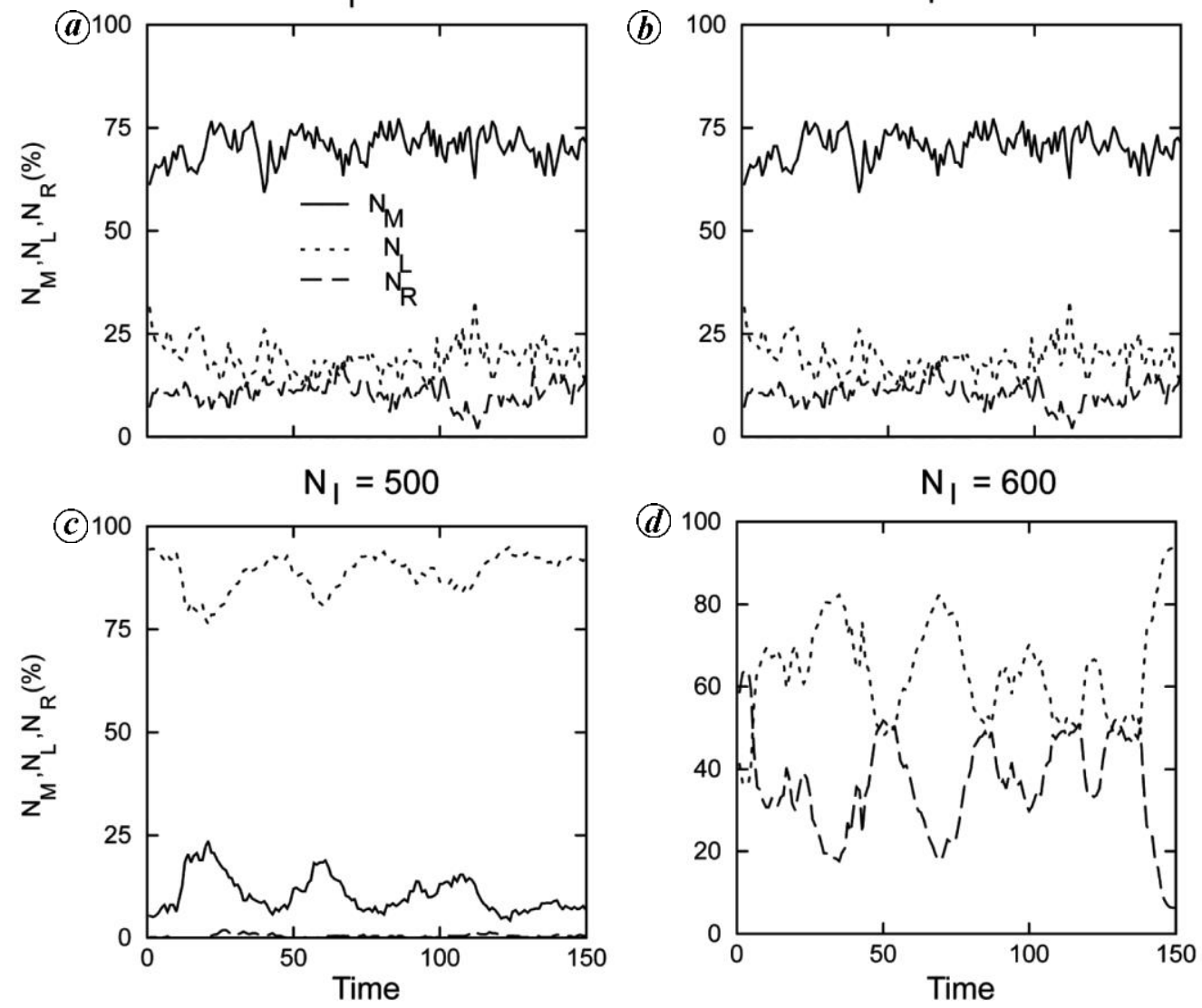

Figure 6. Impact of size of population on growth of membership to prescribed opinion (represented here by the average of individual opinions). Growth of members $\left(N_{\mathrm{M}}(t)\right.$, solid line), extreme leftists $\left(N_{\mathrm{L}}(t)\right.$, dotted line) and extreme rightists $\left(N_{\mathrm{R}}(t)\right.$, long dashed line) as percentage of the total number. Results are shown for four three different values of the total population: 50 (top left panel), 150 (top right panel), 500 (bottom left panel) and 600 (bottom right panel). Values of the parameters correspond to the standard set. $a-d$ represent the subplots of figure.

Table 3. Summary of opinion formation for different scenarios

\begin{tabular}{|c|c|c|c|c|c|}
\hline \multirow[b]{2}{*}{$\begin{array}{l}\text { Scenarios } \\
\text { (prescribed opinion) }\end{array}$} & \multirow[b]{2}{*}{ Scenarios } & \multicolumn{3}{|c|}{ Number (percentage of $N_{\mathrm{I}}$ ) } & \multirow[b]{2}{*}{ Characteristics of evolution (150 years) } \\
\hline & & $\begin{array}{l}\text { Members } \\
\quad\left(N_{\mathrm{M}}\right)\end{array}$ & $\begin{array}{l}\text { Leftists } \\
\qquad\left(N_{\mathrm{L}}\right)\end{array}$ & $\begin{array}{l}\text { Rightists } \\
\qquad\left(N_{\mathrm{R}}\right)\end{array}$ & \\
\hline \multirow[t]{3}{*}{ Average opinion $(\bar{X})$} & $\mathrm{SC} 1$ & 66 & 24. & 9 & \multirow[t]{3}{*}{ Nearly constant } \\
\hline & $\mathrm{SC} 2$ & 70 & 20 & 10 & \\
\hline & $\mathrm{SC} 3$ & 68 & 22 & 9 & \\
\hline \multirow[t]{3}{*}{ Stagnant opinion $\left(X_{\mathrm{I}}\right)$} & SC1 & 65 & 16 & 18 & \multirow{3}{*}{ Strong decrease (increase) in $N_{\mathrm{M}}\left(N_{\mathrm{L}}\right)$ around $t=100$ years } \\
\hline & $\mathrm{SC} 2$ & 63 & 20 & 16 & \\
\hline & SC3 & 68 & 11 & 21 & \\
\hline \multirow[t]{3}{*}{ Maximum opinion $\left(X_{\max }\right)$} & $\mathrm{SC} 1$ & 0 & 62 & 38 & \multirow[t]{3}{*}{ Nearly constant } \\
\hline & $\mathrm{SC} 2$ & 0 & 48 & 52 & \\
\hline & $\mathrm{SC} 3$ & 0 & 57 & 41 & \\
\hline \multirow{2}{*}{ Minimum opinion $\left(X_{\min }\right)$} & $\mathrm{SC} 2$ & 70 & 19 & 10 & \multirow{2}{*}{ High-frequency oscillations in $N_{\mathrm{L}}$ and $N_{\mathrm{R}}$} \\
\hline & SC3 & 76 & 11 & 12 & \\
\hline \multirow[t]{3}{*}{ Random opinion $\left(X_{\mathrm{R}}\right)$} & $\mathrm{SC} 1$ & 13 & 15 & 72 & \multirow[t]{3}{*}{ High-frequency oscillations in $N_{\mathrm{M}}, N_{\mathrm{L}}$ and $N_{\mathrm{R}}$} \\
\hline & $\mathrm{SC} 2$ & 18 & 14 & 68 & \\
\hline & SC3 & 20 & 9 & 71 & \\
\hline
\end{tabular}



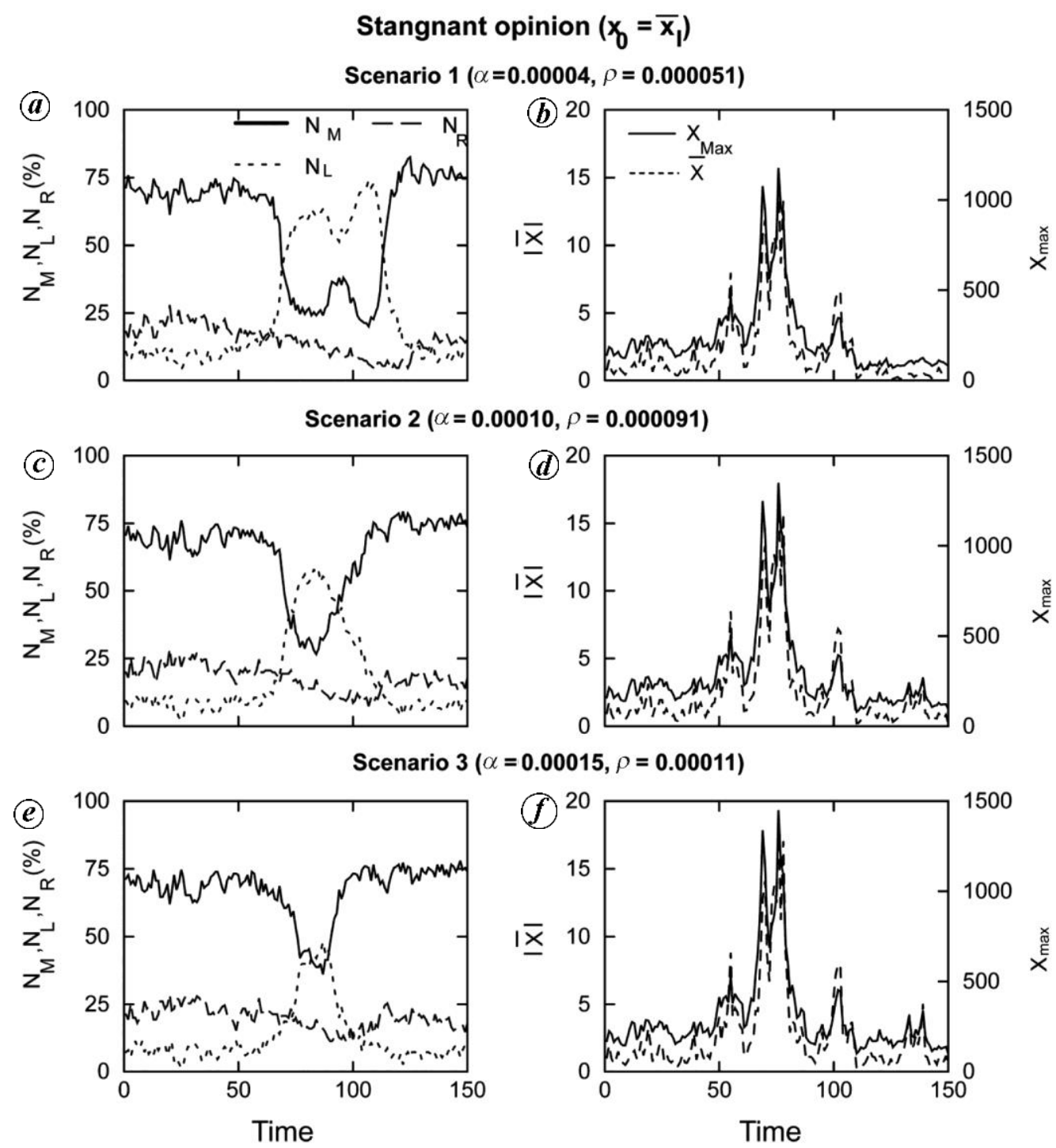

Figure 7. Evolution of membership and characteristics to a prescribed opinion expressed as average of stagnant opinion (average of initial individuals opinions). (Left) Growth of members $\left(N_{\mathrm{M}}(t)\right.$, thick solid line), extreme leftists $\left(N_{\mathrm{L}}(t)\right.$, thin solid line) and extreme rightists $\left(N_{\mathrm{R}}(t)\right.$, dotted line) as percentage of the total number (150). (Right) Average $(\bar{x}(t)$, dashed line, left $y$-axis) and maximum of individual opinions ( $X_{\max }$, solid line, right $y$-axis). Results are shown for three different scenarios in terms of restoration of individual opinion to stagnant opinion $(\alpha)$ and restoration of individual opinion to global opinion $(\rho) . a-f$ represent the subplots of figure.

election or a rise of terrorism are affected by genesis and growth of opinion. Our work shows that causal dynamical models that mimic many characteristics of such opinion dynamics are possible. While the processes and coefficients that drive the model have been chosen for the adopted reference state, the results are not unduly dependent on the variation of these coefficients; in particular, even large $(>100 \%)$ variations in the coefficients produce bounded and meaningful results.

While we have considered many scenarios, the time evolution of membership essentially follows two patterns: nearly constant in time (such as for average opinion or maximum opinion), or high-frequency oscillations, especially in $N_{\mathrm{L}}(t)$ and $N_{\mathrm{R}}(t)$ (Table 3 ). It is noteworthy, however, that strong membership does not evolve for maximum opinion or random opinion (Table 3). In general, the model shows the ability to respond differentially to various prescribed opinions.

It is interesting to note that neither an extreme conservative group $\left(x_{0}=X_{\mathrm{R}} \neq X_{\mathrm{L}}\right)$ nor a group driven by opinions random in time, can lead to significant membership; both result in extreme rightists or extreme leftists. A stagnant prescribed opinion based on the average initial individual opinions fails to create a sustained membership. The present results do not appear sensitive to the global opinion; however, they depend on a number of factors. In particular, we have not considered the representation of global opinion in terms of many 

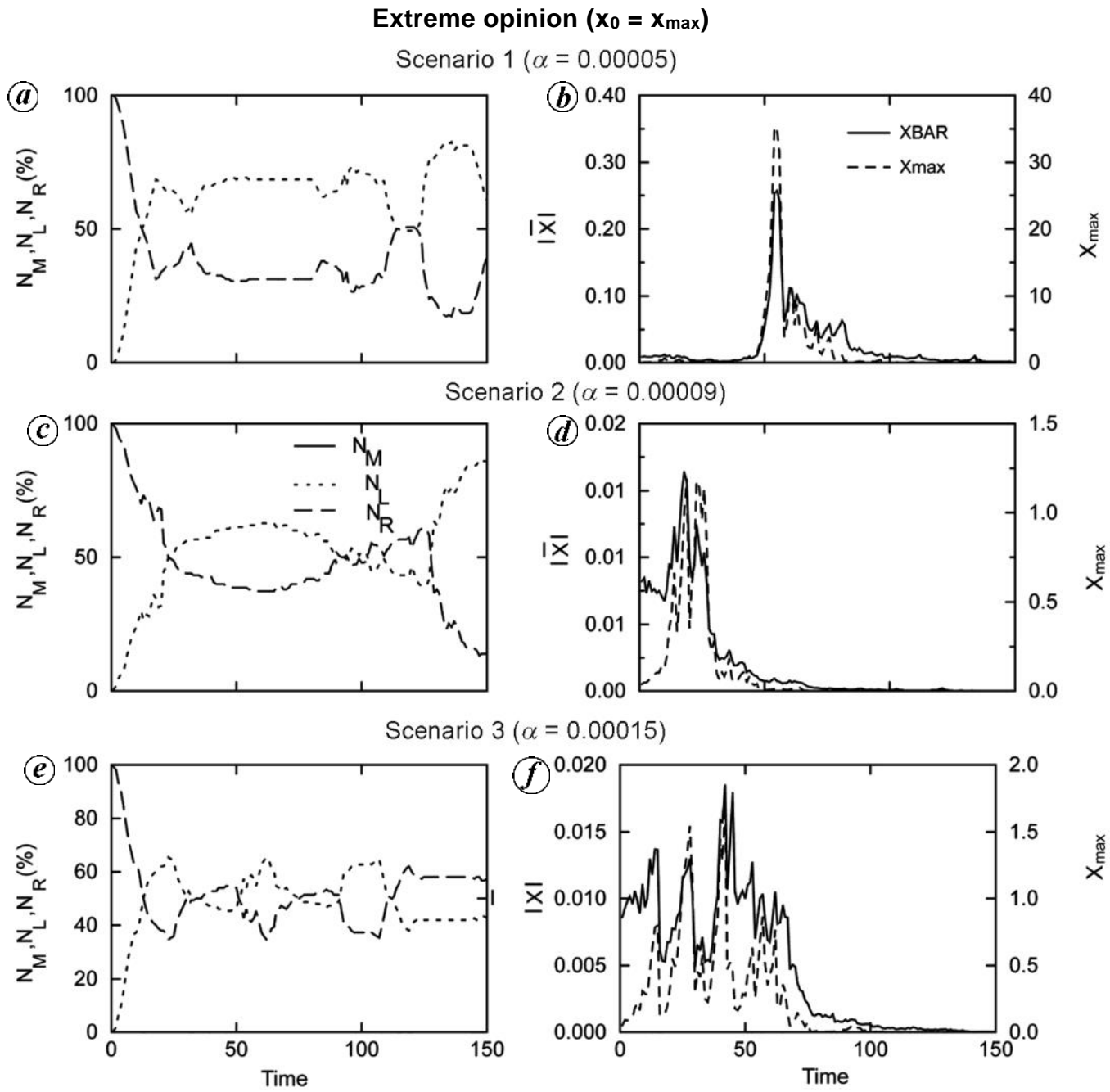

Figure 8. Evolution of membership and characteristics of an opinion expressed as the maximum of individual opinions $\left(X_{\max }\right)$ as a function of time. (Left) Growth of members $\left(N_{\mathrm{M}}(t)\right.$, solid line), extreme leftists $\left(N_{\mathrm{L}}(t)\right.$, dotted line) and extreme rightists $\left(N_{\mathrm{R}}(t)\right.$, long dashed line) as percentage of the total number (150). (Right) Average ( $\bar{x}$, solid line, left $y$-axis) and maximum of individual opinions ( $X_{\max }$, dashed line, right $y$-axis). Results are shown for three different scenarios. Values of the other parameters correspond to the standard set.

processes. The influence of global opinion will also depend on its likely socio-economic impact on the members.

The standard procedure for parameter choice is through calibration and validation based on comparison with observation. Validation, or calibration of our model requires observed data on two groups (control and test) subjected to a given opinion; the test group must be unaware of the externality of the opinion. Such observations are not available and difficult to obtain. In the absence of observed data on opinion dynamics, we need to consider effects where intuitively logical results can be expected to study the behaviour of the system. To our knowledge, there are no comparable studies.

One of the applications of the model can be to examine growth of opinion under different scenarios, such as strength of restoration of individual opinion to prescribed opinion (through state or institutional machinery). It was found that the evolution of membership could respond strongly and in different ways to such scenarios (Figure $4 d-f)$. A particular application of the model can be opinion formation in complex social networks, using methods like Shannon's Entropy ${ }^{38}$ or graph entropy for special weighted graphs ${ }^{39}$. More sophisticated and recent developments like distance-based graph entropies with information functional $^{40}$ can provide new avenues. Undoubtedly, prediction of community (or opinion) evolution in social networks will be an important future direction $^{41}$. An important feature of the present work is that it provides a predictive model of opinion formation as an initial value dynamical system ${ }^{42}$. This opens up a new direction of research in social dynamics.

It needs to be emphasized that our focus has been on a generic model rather than on a specific process. As an 


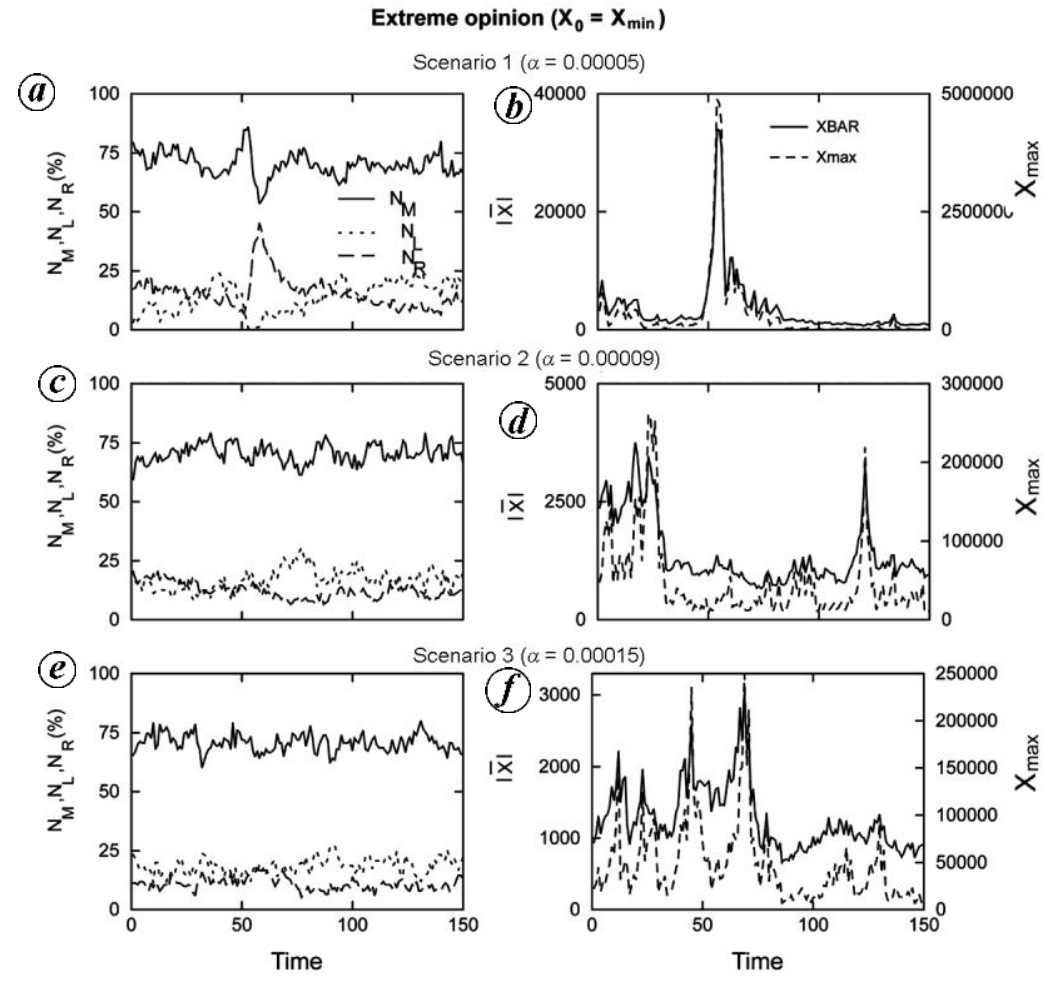

Figure 9. Evolution of membership and characteristics of a prescribed opinion expressed as the minimum of individual opinions $\left(X_{\min }\right)$ as a function of time. (Left) Growth of members $\left(N_{\mathrm{M}}(t)\right.$, solid line), extreme leftists $\left(N_{\mathrm{L}}(t)\right.$, dotted line) and extreme rightists $\left(N_{\mathrm{R}}(t)\right.$, long dashed line) as percentage of the total number (150). (Right) Average $\left(\bar{x}\right.$, solid line, left $y$-axis) and maximum of individual opinions $\left(X_{\max }\right.$, dashed solid line, right $y$-axis). Results are shown for three different scenarios. Values of the other parameters correspond to the standard set.

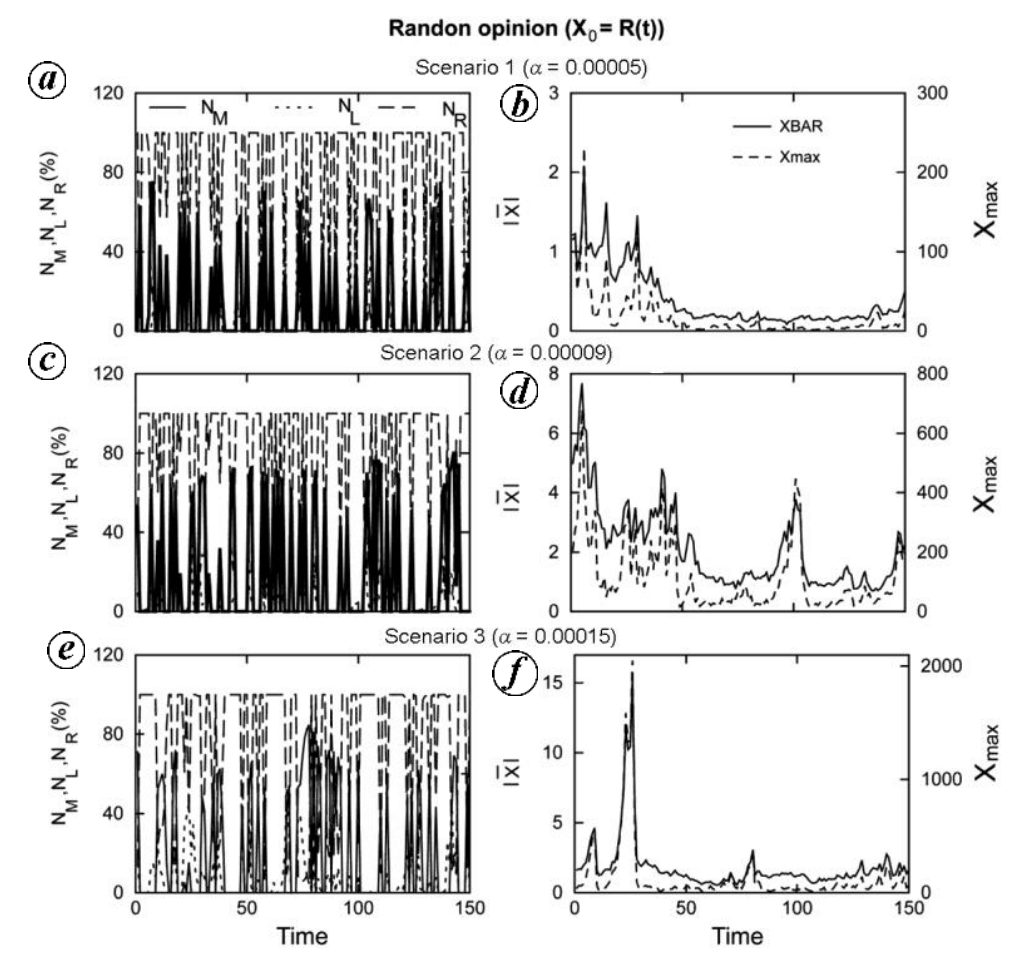

Figure 10. Evolution of membership and characteristics of a prescribed opinion expressed as a random variable as a function of time. (Left) Growth of members $\left(N_{\mathrm{M}}(t)\right.$, solid line), extreme leftists $\left(N_{\mathrm{L}}(t)\right.$, dotted line) and extreme rightists $\left(N_{\mathrm{R}}(t)\right.$, long dashed line) as percentage of the total numbers (150). (Right) Average ( $\bar{x}$, solid line, left $y$-axis) and maximum of individual opinions ( $X_{\max }$, dashed line, right $y$-axis). Results are shown for three different scenarios. Values of the other parameters correspond to the standard set. 
Average opinion $\left(\mathrm{X}_{0}=\overline{\mathbf{X}}(\mathrm{t})\right)$
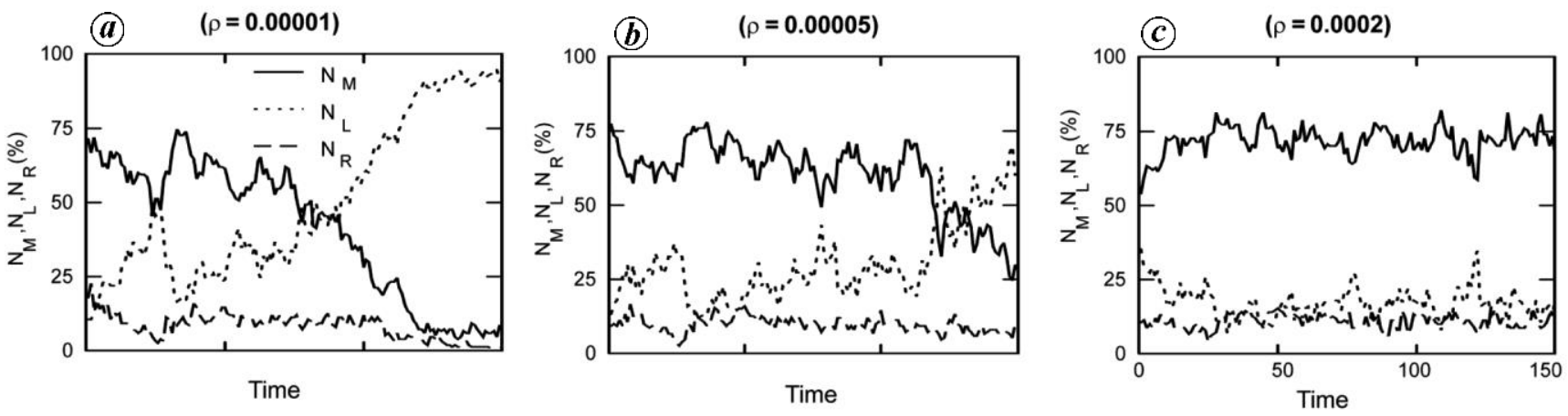

Figure 11. Impact of strength of restoration of average of individual opinion $\left(x_{0}\right)$ to global opinion. Values of the other parameters correspond to the standard set.
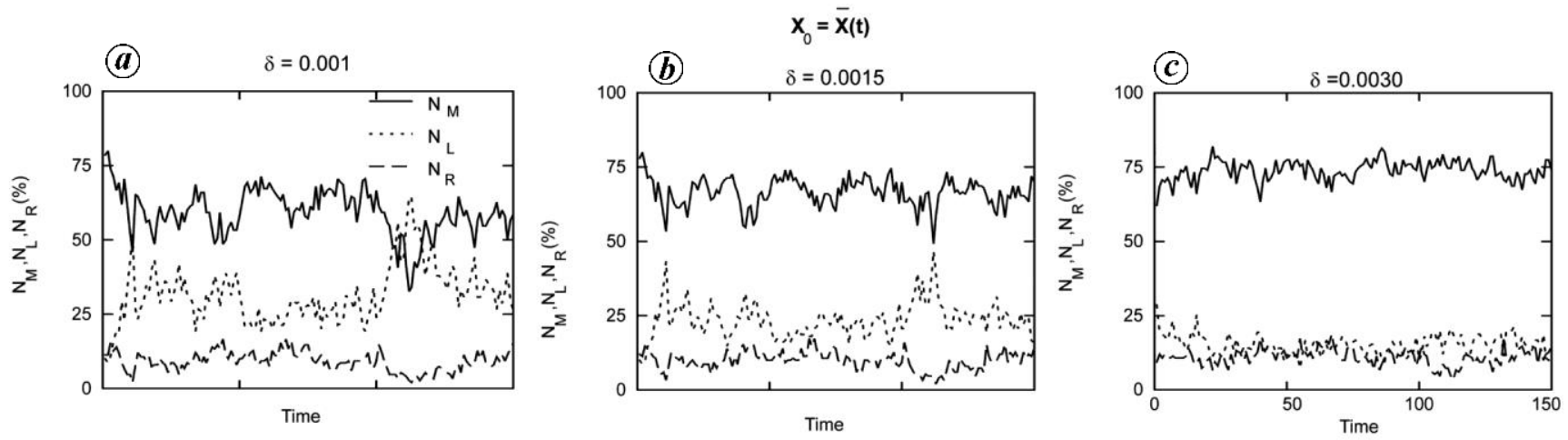

Figure 12. Impact of strength of restoration of the prescribed opinion to the global opinion on the growth of membership; here prescribed opinion is represented by the average opinion of the population. Values of the other parameters correspond to the standard set.

analogy, our equation represents a general dynamics of fluids; the initial, boundary conditions as well as the specific forcings, and sources and sinks will depend on the specifics of the process. It is assumed that the response of an individual can be represented as a scalar that combines economic, cultural and emotional aspects. Quite clearly, this is a simplification; in the subsequent versions, the response needs to be represented through an appropriate vector. It will be also necessary, and natural as the theory evolves, to include abstract processes into the opinion dynamics ${ }^{17-20}$. For practical applications it will be necessary, but possible, to assign specific values and constraints. Naturally, this will depend on the process being studied as well as the target population. The prescribed opinion can be more specific, such as a policy or an election manifesto. An important area of future work could be the study of dynamics of social processes like rise of terrorism, and possibly laying the foundation for social engineering. In such applications, pre-designed opinion (proposition) can be visualized for goal-oriented growth of membership with the rapidly growing computing power and social networks and such applications are becoming feasible.
1. Jalili, M., Social power and opinion formation in complex networks. Phys. A, 2013, 392(4), 959-966.

2. Hegselmann, R. and Flache, A., Understanding complex social dynamics - a plea for cellular automata based modelling. J. Art. Soci. Soc. Simul., 1998, 1.

3. Li, P. P. and Hui, P. M., Dynamics of opinion formation in hierarchical social networks: networks structure and initial bias. Eur. Phys. J.B, 2008, 61, 371-376.

4. Toral R. and Tessone, C. J., Finite size effects in the dynamics of opinion formation. Commun. Comput. Phys., 2007, 2, 177-195.

5. Wu, F. and Huberman, B., Social structure and opinion formation. Comput. Econ., 2004, 0407002.

6. Moussaid, M., Kammer, J. E., Analytis, P. P. and Neth, H., Social influence and the collective dynamics of opinion formation. PLoS ONE, 2013, 8(11), e78433.

7. Krause, U., A discrete nonlinear and non-autonomous model of consensus formation. In Communications in Difference Equations (eds Flaydi, S. et al.), Gordon and Breach Publication, Amsterdam, 2000, pp. 227-236.

8. Shang, Y., Consensus formation of two-level opinion dynamics. Acta Math. Sci., 2014, 34(4), 1029-1040.

9. Takao, F., A simple model of consensus formation. Okayama Econ. Rev., 1999, 31, 95-100.

10. Galam, S., The dynamics of minority opinions in democratic debate. Phys. A, 2004, 336, 56-62.

11. Laguna, M. F., Abramson, G. and Zanette, D., Minorities in a model for opinion formation. Complexity, 2004, 9(4), 31-36.

CURRENT SCIENCE, VOL. 116, NO. 4, 25 FEBRUARY 2019 
12. Mogilner, A., Edelstein-Keshet, L., Bent, L. and Spiros, A., Mutual interactions, potentials, and individual distance in a social aggregation. J. Math. Biol., 2003, 47, 353-389.

13. Mobilia, M. and Redner, S., Majority versus minority dynamics: phase transition in an interacting two-state spin system. Phys. Rev. E, 2003, 68(4), 046106.

14. Boccara, N., Models of opinion formation: influence of opinion leaders. Int. J. Mod. Phys. C, 2008, 19, 93-109.

15. Crespi, B. J., The evolution of social behaviour in microorganisms. Trends Ecol. Evol., 2001, 16(4), 178-183.

16. Wollman, N. P. et al., The dynamics of animal social networks: analytical, conceptual, and theoretical advances. Behav. Ecol., 2013, 25(2), 242-255.

17. Ghosh, R. and Lerman, K., The impact of network flows on community formation in models of opinion dynamics. J. Math. Soc., 2015, 39(2), 109-124.

18. Hoylst, J. A., Kacperski, K. and Schweitzer, F., Social impact models of opinion dynamics. Ann. Rev. Comput. Phys., 2001, IX, 253-273.

19. Antal, T., Krapivsky, P. L. and Redner, S., Dynamics of social balance on networks. Phys. Rev. E, 2005, 72, 036121.

20. Altafini, C., Dynamics of opinion forming in structurally balanced social networks. PLoS ONE, 2012, 7(6), e38135.

21. Gueron, S., Levin, S. A. and Rubenstein, D. I., The dynamics of herds: from individuals to aggregations. J. Theor. Biol., 1996, 182(1), 85-98.

22. Fieldhouse, E., Shryane, N. and Pickles, A., Strategic voting and constituency context: modelling party preference and vote in multiparty elections. Polit. Geogr., 2007, 26(2), 159-178.

23. Jiao, Y., Syau, Y. R. and Lee, E. S., Fuzzy adaptive network in presidential elections. Math. Comput. Model., 2006, 43, 244-253.

24. Berger, R. L., A necessary and sufficient condition for reaching a consensus using DeGroot's method. J. Am. Stat. Assoc., 1981, 76(374), 415-418.

25. Kosse, K., Group size and societal complexity: thresholds in the long-term memory. J. Anthropol. Archaeol., 1990, 9, 275-303.

26. Farine, D. R. et al., The role of social and ecological processes in structuring animal populations: a case study from automated tracking of wild birds. R. Soc. Open Sci., 2015, 2, 150057.

27. Belenky, A. S. and King, D. C., A mathematical model for estimating the potential margin of state undecided voters for a candidate in a US Federal election. Math. Comput. Model., 2007, 45, 585-593.

28. Mishra, A. K., A simple mathematical model for the spread of two political parties. Nonlinear Anal. Model. Control, 2012, 17, $343-354$
29. Farley, J. D., Evolutionary dynamics of the insurgency in Iraq: a mathematical model of the battle for hearts and minds. Stud. Confl. Terror., 2007, 30(11), 947-962.

30. Anderton, C. H. and Carter, J. R., On rational choice theory and the study of terrorism. Def. Peace Econ., 2005, 16, 275-282.

31. Facchetti, G., Iacono, G. and Altafini, C., Computing global structural balance in large-scale signed social networks. Proc. Natl. Acad. Sci., 2011, 108(52), 20953-20958.

32. Latane, B. and Nowak, A., Self-organizing social systems, necessary and sufficient conditions for the emergence of clustering, consolidation, and continuing diversity. In Progress in Communication Science: Advances in Persuasion (eds Barnet, G. and Bostner, F.), Norwood, NJ, USA, Ablex Publishing Corporation, 1997, pp. 43-74.

33. Iacono, G. and Altafini, C., Monotonicity, frustration, and ordered response: an analysis of the energy landscape of perturbed largescale biological networks. BMC Syst. Biol., 2010, 4(1), 83.

34. Weisbuch, G., Deffuant, G. and Amblard, F., Persuasion dynamics. Phys. A, 2005, 353, 555-575.

35. Kawachi, K., Deterministic models for rumor transmission. Nonlinear Anal. Real World Appl., 2008, 9, 1989-2028.

36. Hegselmann, R. and Krause, U., Opinion dynamics and bounded confidence: models, analysis, and simulation. JASSS, 2002, 5(3), $1-33$.

37. Klir, G. J. and Yuan, B., Fuzzy Sets and Fuzzy Logic; Theory and Applications, Prentice Hall PTR, New Jersey, 1995.

38. Cao, S., Dehmer, M. and Shi, Y., Extremality of degree-based graph entropies. Inf. Sci., 2014, 278, 22-33.

39. Chen, Z., Dehmer, M., Emmert-Streib, F. and Shi, Y., Entropy of weighted graphs with Randi'c weights. Entropy, 2015, 17(6), 3710-3723.

40. Chen, Z., Dehmer, M. and Shi, Y., A note on distance-based graph entropies. Entropy, 2014, 16(10), 5416-5427.

41. Chen, Z., Dehmer, M. and Shi, Y., Bounds for degree-based network entropies. Appl. Math. Comput, 2015, 265, 983-993.

42. Saganowski, S. et al., Predicting community evolution in social networks. Entropy, 2015, 17(5), 3053-3096.

ACKNOWLEDGEMENTS. This work was supported by CSIR, New Delhi. The authors declare no conflict of interest. S.N. thanks the Department of Mathematics, Ramaiah University of Applied Sciences, Peenya Campus, Bengaluru for support.

Received 3 January 2017; revised accepted 19 September 2018 doi: $10.18520 / \mathrm{cs} / \mathrm{v} 116 / \mathrm{i} 4 / 577-591$ 